\title{
Measurement of End-Loss Ions Originated from Spontaneously Excited High Frequency Waves by Using an MCP Detector on GAMMA 10/PDX ${ }^{*}$
}

\author{
Koki IZUMI, Ryuya IKEZOE ${ }^{1)}$, Makoto ICHIMURA, Mafumi HIRATA, \\ Mizuki SAKAMOTO, Shuhei SUMIDA, Seowon JANG, Atsuto TANAKA, \\ Yushi KUBOTA, Ryo SEKINE, Hiroki KAYANO and Yousuke NAKASHIMA \\ Plasma Research Center, University of Tsukuba, Tsukuba 305-8577, Japan \\ ${ }^{1)}$ Research Institute for Applied Mechanics, Kyushu University, Kasuga 816-8580, Japan
}

(Received 30 September 2018 / Accepted 9 January 2019)

\begin{abstract}
Effective ICRF heating creates a high ion-temperature plasma with strong ion-temperature anisotropy in the GAMMA 10/PDX central cell, where several Alfvén-ion-cyclotron (AIC) waves are spontaneously excited. It is clearly measured with a microwave reflectometer installed in the central cell that waves with the difference frequencies of the AIC waves are excited in a core region. In addition, it has been observed with an end-loss high-energy ion detector that high-energy ions of over $6 \mathrm{keV}$ are axially transported along the magnetic field lines with the same frequencies as the difference frequencies of the AIC waves. In this study, in order to understand the related wave-particle interaction, another end-loss high-energy ion detector using a micro-channel plate has been developed. By changing the ion retarding grid voltage, which the new detector equips, it is found that an energy dependence exists in the loss mechanism; some frequencies that the axial transport includes disappear for high-energy ions of over $6 \mathrm{keV}$ while they are significant for low-energy ions. In addition, by changing the radial position of the new detector, a radial localization of the axial transport to a core region, which is consistent with the profile of the difference frequencies waves, is indicated.
\end{abstract}

(c) 2019 The Japan Society of Plasma Science and Nuclear Fusion Research

Keywords: GAMMA 10/PDX, high-energy ion, Alfvén-ion-cyclotron wave, low-frequency wave, wave-particle interaction

DOI: $10.1585 /$ pfr. 14.2402033

\section{Introduction}

In the main confinement region, called central cell, of the GAMMA 10/PDX tandem mirror, ion temperature in the perpendicular direction to the magnetic field line, $T_{\mathrm{i} \perp}$, reaches several $\mathrm{keV}$ owing to ion cyclotron range of frequencies (ICRF) heating. The ion temperature anisotropy which is defined as the ratio of $T_{\mathrm{i} \perp}$ to $T_{\mathrm{i} \|}$ becomes more than 10 , where $T_{\mathrm{i} \|}$ is the ion temperature in the parallel direction to the magnetic field line. Under such conditions, a slow Alfvén wave called as the Alfvén-Ion-Cyclotron (AIC) wave is spontaneously excited just below the ion cyclotron frequency [1]. Figure 1 shows a frequency spectrum of the density fluctuation measured with a microwave reflectometer in the central cell of GAMMA 10/PDX. A peak at $6.36 \mathrm{MHz}$ is the applied frequency for ion heating in the central cell. The peaks appear below $6.0 \mathrm{MHz}$ are the AIC waves. They are labeled as AIC1 to AIC4 in Fig. 1 to refer in this paper. The AIC waves emerge in the unstable frequency range as discrete peaks and the difference frequencies (DF) between their neighboring peaks are around

author'se-mail: izumi_koki@prc.tsukuba.ac.jp

*) This article is based on the presentation at the 12th International Conference on Open Magnetic Systems for Plasma Confinement (OS2018).

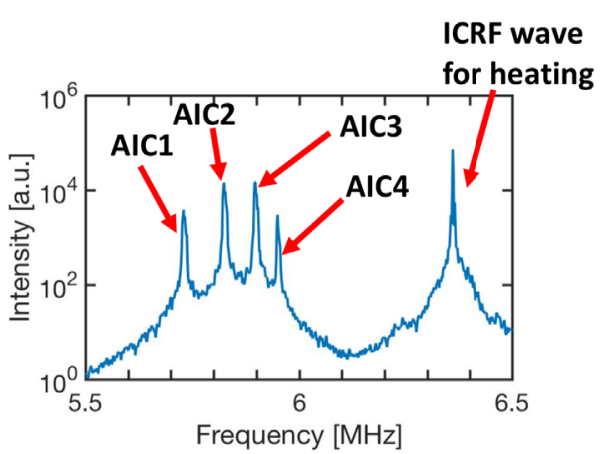

Fig. 1 A typical frequency spectrum of the density fluctuation measured by a microwave reflectometer in the central cell.

$100 \mathrm{kHz}$.

It is also clearly measured with a microwave reflectometer in the central cell that DF waves are excited by nonlinear couplings among the AIC waves especially in the core region [2]. We call an inner region inside the half plasma radius of $9 \mathrm{~cm}$ as the core region in the following for convenience. In addition, it has been observed with an east end-loss high-energy ion detector using a semi- 
conductor (eeHED) that high-energy ions are periodically transported to the end along the magnetic field lines with the same frequencies as the DF of the AIC waves [3]. In this study, we have developed an east end-loss high-energy ions detector using a micro-channel plate (eeMCP) with an electrostatic energy analyzer, and measured axially transported high-energy ions at two radial positions by changing the installation port. The radial and energy dependences of axial transport of high-energy ions that is caused by the DF waves are investigated using the eeMCP.

\section{GAMMA 10/PDX and ICRF Sys- tems}

GAMMA 10/PDX is a tandem mirror plasma confinement device and consists of 5 mirror cells and end regions [4]. There are three ICRF systems in GAMMMA 10/PDX called RF1, RF2 and RF3. RF1 and RF2 have two sets of power amplifiers, and RF3 has a single set. Two Nagoya Type-III (Type-III) antennas and two double half turn (DHT) antennas are installed in the central cell of GAMMA 10/PDX.

In this study standard discharges were used, where a seed plasma was injected from the plasma gun at the east end of the machine and fast waves of $9.9 \mathrm{MHz}$ and 10.3 MHz were excited by the Type-III antennas with RF1, and slow wave of $6.36 \mathrm{MHz}$ was excited by DHT antennas with RF2. The fast waves produce plasma and heat ions near the midplane of both anchor cells [5]. The slow waves heat ions near the midplane of the central cell. RF3 can be used for several purposes while in this study it is not used, instead, a sub-pulse is used for RF2, which changes the RF power with a step-like waveform.

\section{Diagnostics}

Figure 2 shows schematic drawings of the eeMCP; (a) whole device, (b) the electrostatic energy analysis unit and (c) the electrostatic potential profile. A micro-channel plate (MCP) (F4655-10; Hamamatsu Photonics K.K.) is used for the detector of the eeMCP. Collimators are installed to limit the number of incident ions and prevent saturation of MCP; the diameters of the variable collimator located just before the entrance of the energy analyzer are $0.5 \mathrm{~mm}, 2.0 \mathrm{~mm}$ and $8.0 \mathrm{~mm}$. In the present experiments, $8.0 \mathrm{~mm}$ is used, which is a full open setting. In this case, the entrance hole of the energy analyzer determines the collimator diameter as $4 \mathrm{~mm}$. These collimators are also utilized for eliminating an intense light from the plasma along with their unique installation configuration; eeMCP is installed at the east end of the machine along the magnetic field line at there, which is inclining to the magnetic axis due to the open field configuration. Therefore, the view sight of the MCP does not include the dense plasma region such as central and anchor plasma.

The energy analyzer consists of two electrostatic grids (a)

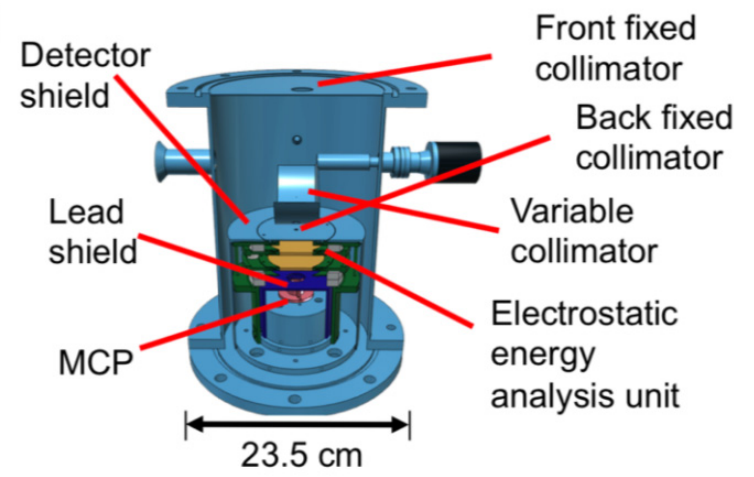

(b)

Back fixed

b) collimator

(c)
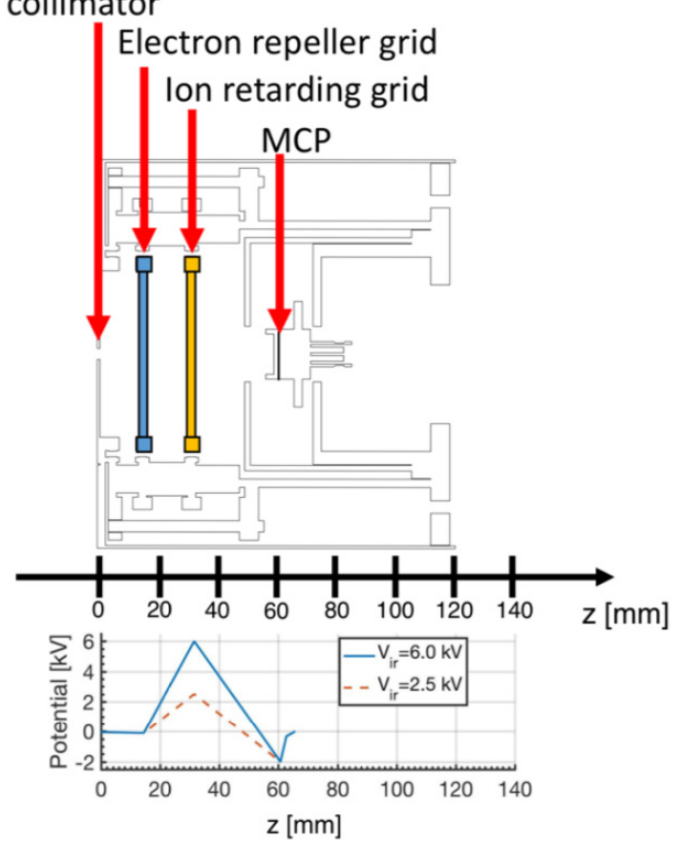

Fig. 2 Schematic drawings of the eeMCP; (a) whole device, (b) the electrostatic energy analysis unit and (c) the electrostatic potential profile.

for electron repeller and ion retarding grids along with grids for MCP. The voltage on the electron repeller grid is $-100 \mathrm{~V}$; electron temperature is a few tens $\mathrm{eV}$. Ion retarding voltage, $V_{\text {ir }}$, is varied up to $7 \mathrm{kV}$, which determines the minimum energy of detecting ions. A few incident electrons with energies higher than the electron repeller voltage are retarded in front of the MCP, where acceleration voltage of about $-2 \mathrm{kV}$ is applied.

The radial position of the eeMCP can be selected to $r_{\mathrm{cc}} \sim 4 \mathrm{~cm}$ or $r_{\mathrm{cc}} \sim 10 \mathrm{~cm}$ using two available ports, where $r_{\mathrm{cc}}$ is the radius converted into that at the midplane in the central cell along the same field line.

\section{Experimental Results}

\subsection{Comparison of eeMCP and eeHED}

We have successfully measured signals of end-loss high-energy ions which fluctuated with the frequencies of 


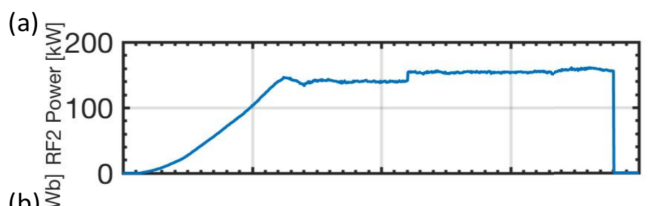

(b)

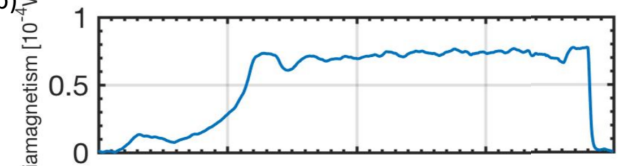

(c) $\stackrel{\frac{\sqrt{6}}{a}}{\frac{2}{4}}$

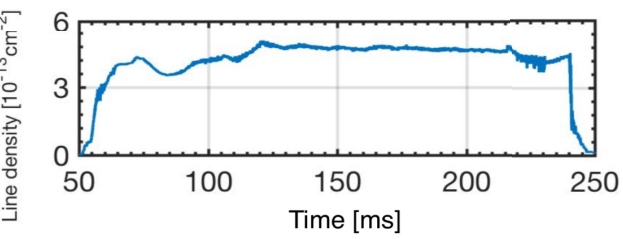

Fig. 3 Time evolution of (a) input power of RF2, (b) diamagnetism and (c) line density near the midplane of the central cell in a typical discharge.

the DF waves by using the eeMCP. The pulses that the MCP generates in response to incident ions are well pileupped, and thus an eeMCP signal, or current, is related to the total incident ions flux. Here, at first, we compare an eeMCP signal to an established ion signal of the eeHED.

Figure 3 shows the time evolution of (a) the input power of RF2, (b) the diamagnetism and (c) the line density near the midplane of the central cell for one of the discharges used in this study. The power of RF2 is raised till $113 \mathrm{~ms}$ and, after the following steady-period, it is slightly increased with a sub-pulse from $160 \mathrm{~ms}$ to the end of the discharge $240 \mathrm{~ms}$. In this study, by varying the sub-pulse power, signals of the eeMCP were taken for a wide power range of the AIC waves, where the power of DF waves should be varied as well. After the rump-up of RF2, the line density does not vary much with the variation in the RF2 power since the density is sustained mostly by RF1 system. In the discharge shown in Fig. 3, an ECH is applied to the central plasma from $215 \mathrm{~ms}$, of which period is not used for the analyses shown in the following.

Figure 4 shows the time evolution of the frequency spectrum of the density fluctuation measured by a microwave reflectometer and end-loss high-energy ions signals measured by the eeMCP and the eeHED in the same discharge. The $V_{\text {ir }}$ of the eeMCP is set to be $6 \mathrm{kV}$, which is the minimum detection energy of the eeHED. The radial position of both detectors is the same, about $r_{\mathrm{cc}}=$ $10 \mathrm{~cm}$. In Fig. 4 , the notation $f_{(\mathrm{AIC} 2-\mathrm{AIC} 1)}$ means the difference frequency between AIC2 and AIC1. Comparing the eeMCP with the eeHED signals, though there are slight differences, similar time evolution is obtained as expected. When the radius and the minimum detection ion energy of the eeMCP were different, much different behavior was obtained, which are shown below.
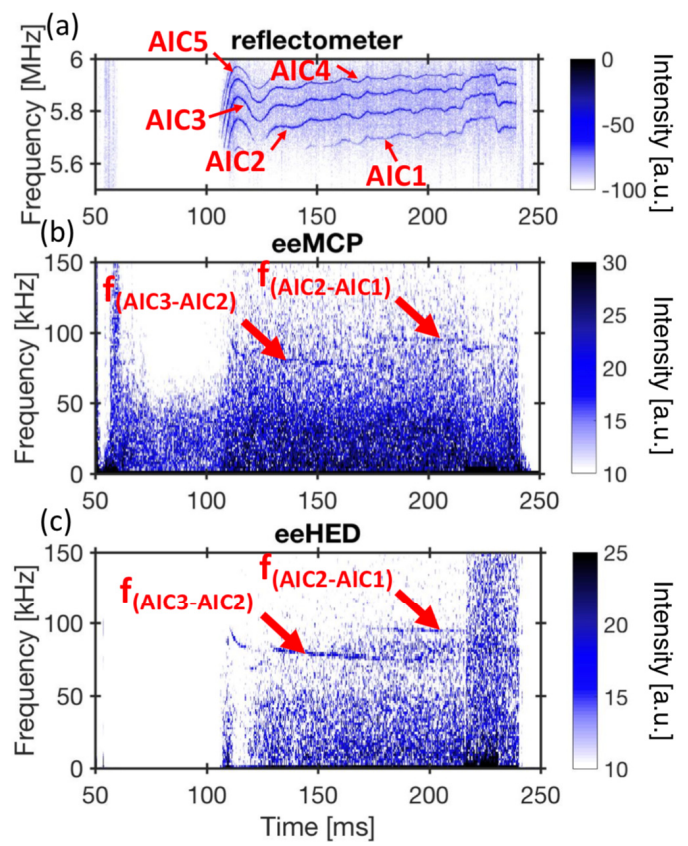

Fig. 4 Time evolution of the frequency spectrum of (a) density fluctuation measured by a microwave reflectometer and (b) end-loss high-energy ions with energies above $6 \mathrm{keV}$ measured by the eeMCP and (c) the eeHED. Both highenergy ion detectors are installed at $r_{\mathrm{cc}} \sim 10 \mathrm{~cm}$.

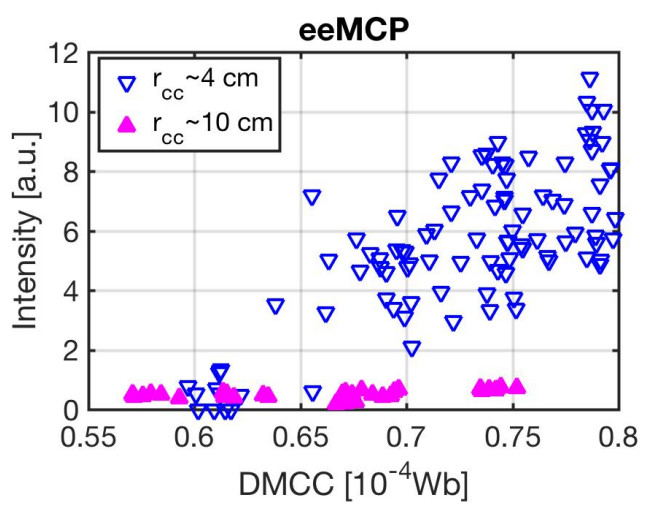

Fig. 5 Comparison of the intensity of the difference frequency fluctuations measured by the eeMCP at different radius of $r_{\mathrm{cc}} \sim 4$ and $10 \mathrm{~cm}$ as a function of the diamagnetism near the midplane in the central cell.

\subsection{Radial dependence of end-loss high- energy ions with difference frequency fluctuations}

Figure 5 compares the intensity of the DF fluctuations measured by the same eeMCP with $V_{\text {ir }}$ of $6.0 \mathrm{kV}$ but at different radial positions of about $r_{\mathrm{cc}}=4$ and $10 \mathrm{~cm}$ as a function of the diamagnetism near the midplane of the central cell (DMCC). The plasma conditions before the sub-pulse of RF2 are adjusted to be identical for all the discharges analyzed here. It is clearly shown that the intensities of the DF fluctuation at $r_{\mathrm{cc}} \sim 4 \mathrm{~cm}$ are much higher than that at 

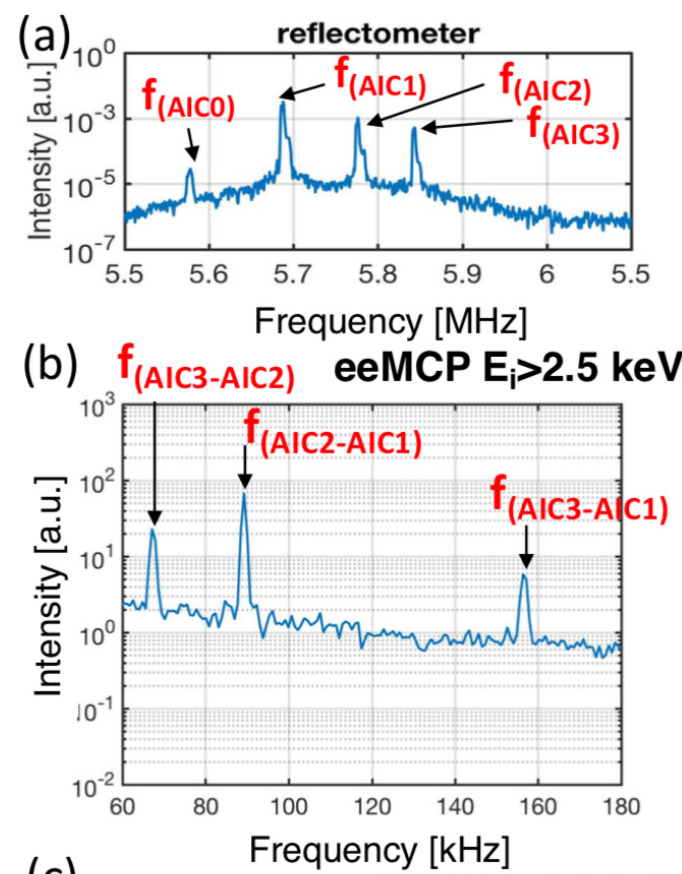

(c)

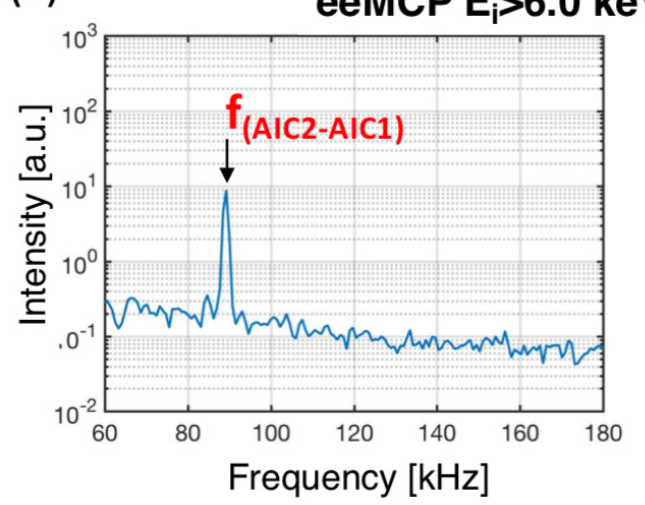

Fig. 6 Frequency spectrum of (a) density fluctuation measured by a microwave reflectometer and (b) end-loss highenergy ions with energy above $2.5 \mathrm{keV}$ and (c) $6.0 \mathrm{keV}$ measured by the eeMCP installed at $r_{\mathrm{cc}} \sim 4 \mathrm{~cm}$.

$r_{\mathrm{cc}} \sim 10 \mathrm{~cm}$.

Although the measurements were conducted in different discharges to change the installation port of the eeMCP, the comparison is valid since the same DMCC under a similar plasma condition supports a similar behavior of the AIC and DF waves. Therefore, above result indicates that the interaction between the DF waves and the high-energy ions, which leads to the axial transport, is significant near core. It should be noted that a realistic ion temperature and density profile in the radial direction cannot change the above statement. One of the reason would be the radial localization of the DF waves; the radial intensity profile of the DF waves that are measured by a reflectometer in the central cell indicates that the significant DF waves exist only in a core region inside the half plasma radius of $9 \mathrm{~cm}$.

\subsection{Energy dependence of end-loss high- energy ions}

Figure 6 shows the frequency spectrum of (a) density fluctuation measured by a microwave reflectometer and the end-loss high-energy ions measured by the eeMCP at $r_{\mathrm{cc}} \sim 4 \mathrm{~cm}$ with the ion retarding voltages of (b) $2.5 \mathrm{kV}$ and (c) $6.0 \mathrm{kV}$. In Fig. 6(b), three peaks are clearly observed. The frequencies of these three peaks correspond to the difference frequencies of the AIC waves, that are, AIC3-AIC2, AIC2-AIC1 and AIC3-AIC1. On the other hand, only the peak of AIC2-AIC1 is observed in Fig. 6 (c) even though the plasma parameters are almost same for both discharges. This result suggests that the energy of the transported ions depends on the interacted DF waves.

\section{Summary}

An end-loss high-energy ion detector using an MCP (eeMCP) has been developed and installed at the east end of GAMMA 10/PDX to measure end-loss high-energy ions. The eeMCP can vary the minimum energy of incident ions by using an ion retarding potential, and can change its radial position. The measured high-energy ion signal shows an effect of the waves with the difference frequencies of the AIC waves. The interaction effect is more active near core, which agrees with the radial profile of the difference frequency fluctuations. Furthermore, it is observed that the interaction effect has a dependence on the energy of ions, which is different for different DF waves. More detailed measurement of the energy dependence is a future work.

\section{Acknowledgments}

The authors acknowledge the member of Plasma Research Center in University of Tsukuba for their collaboration. This work was partly supported by the bidirectional collaborative research program of the National Institute for Fusion Science, Japan (NIFS14KUGM086 and NIFS17KUGM132) and JSPS KAKENHI (18K03574).

[1] M. Ichimura et al., Phys. Rev. Lett. 70, 2734 (1993).

[2] R. Ikezoe et al., Phys. Plasmas 22, 090701 (2015).

[3] R. Ikezoe et al., Nucl. Fusion 53, 073040 (2013).

[4] M. Inutake et al., Phys. Rev. Lett. 55, 939 (1985).

[5] M. Ichimura et al., Nucl. Fusion 28, 799 (1988). 